\title{
Métodos de digestão de solos para determinação do teor de vanádio
}

Os métodos para a determinação dos teores de elementos-traço presentes no solo devem proporcionar a exatidão de resultados, baixo custo, praticidade e simplicidade. Objetivou-se neste estudo comparar dois métodos de abertura das amostras, USEPA 3050B e USEPA 3051A, quanto à eficiência de determinação de teores de vanádio no solo. A avaliação foi conduzida com base em amostras de referência NIST 2709A (SRM San Joaquin Soil) e em amostras do horizonte A de classes de solos do estado de Santa Catarina, pertencentes ao banco de dados do Departamento de Solos da UDESC. A abertura das amostras foi procedida utilizando-se os dois métodos supramencionados e a quantificação de vanádio foi efetuada por meio de espectroscopia de emissão atômica por plasma acoplado indutivamente (ICP-OES). Os dados foram analisados por meio de estatística descritiva, analise de correlação e regressão linear, cálculo de coeficientes de concordância e teste de identidade, utilizando-se de planilha eletrônica e software R. Na média, os maiores teores de vanádio nas amostras de referência foram obtidos com o método USEPA 3051A (93,50\%), comparativamente ao método USEPA 3050B $(69,10 \%)$. Nas amostras de solos, os valores dos coeficientes de correlação linear $(0,96)$, de determinação $(0,92)$, do índice de concordância de Willmott $(0,87)$ e do coeficiente de confiança de Camargo e Sentelhas $(0,83)$ indicaram alto grau de associação/concordância entre os dois métodos testados. No entanto, o teste de identidade proposto por Leite e Oliveira indicou que há diferença $(P<0,05)$ quanto à eficiência de determinação dos teores de vanádio em solos entre eles.

Palavras-chave: Elementos-traço; Digestão de amostras; Regressão linear; Concordância; Teste de identidade.

\section{Soil digestion methods for determining the vanadium content}

The methods for determining the levels of trace elements present in the soil must provide the accuracy of results, low cost, practicality and simplicity. The objective of this study was to compare two methods of opening the samples, USEPA 3050B and USEPA 3051A, regarding the efficiency of determining vanadium contents in the soil. The evaluation was conducted based on reference samples NIST 2709A (SRM San Joaquin Soil) and samples from horizon A of soil classes in the state of Santa Catarina, belonging to the UDESC's Soil Department database. The opening of the samples was carried out using the two methods mentioned above and the quantification of vanadium was carried out by means of inductively coupled plasma atomic emission spectroscopy (ICP-OES). The data were analyzed using descriptive statistics, correlation analysis and linear regression, calculation of concordance coefficients and identity test, using an electronic spreadsheet and $\mathrm{R}$ software. On average, the highest vanadium contents in the reference samples were obtained with the USEPA 3051A method (93.50\%), compared to the USEPA 3050B method (69.10\%). In the soil samples, the values of the linear correlation coefficients (0.96), of determination (0.92), Willmott's agreement index (0.87) and Camargo and Sentelhas' confidence coefficient (0.83) indicated a high degree of association/agreement between the two methods tested. However, the identity test proposed by Leite and Oliveira indicated that there is a difference $(P<0.05)$ regarding the efficiency of determining vanadium content in soils between them.

Keywords: Trace elements; Sample digestion; Linear regression; Concordance; Identity test.

Topic: Ciências do Solo

Reviewed anonymously in the process of blind peer.
Received: 02/07/2021

Approved: 27/07/2021
Marcos Roberto Schein (iD)

Universidade do Estado de Santa Catarina, Brasil http://lattes.cnpq.br/2956302527012851

http://orcid.org/0000-0002-2882-1201

shinelo46@hotmail.com

David José Miquelluti (D)

Universidade do Estado de Santa Catarina, Brasil http://lattes.cnpq.br/0683203409889468

http://orcid.org/0000-0002-7369-6163

david.miquelluti@udesc.br

Mari Lucia Campos (ib

Universidade do Estado de Santa Catarina, Brasil

http://lattes.cnpq.br/4291057837136509

http://orcid.org/0000-0003-3250-2067

mari.campos@udesc.br
Ilana Marin Suppi (iD

Universidade do Estado de Santa Catarina, Brasil

http://lattes.cnpq.br/973225533053232

http://orcid.org/0000-0002-5224-2875

ilanalgs@yahoo.com.br
Referencing this:

SCHEIN, M. R.; MIQUELLUTI, D. J.; CAMPOS, M. L.; SUPPI, I. M..

Métodos de digestão de solos para determinação do teor de vanádio. Revista Ibero Americana de Ciências Ambientais, v.12, n.7, p.53-60, 2021. DOI: http://doi.org/10.6008/CBPC2179-6858.2021.007.0005

DOI: 10.6008/CBPC2179-6858.2021.007.0005 


\section{INTRODUÇÃO}

O emprego de diferentes métodos analíticos para a determinação de elementos-traço em plantas e solos tem proporcionado instrumentos necessários tanto para o gerenciamento de riscos a que estão sujeitos a população e o meio ambiente, quanto na tomada de decisões referente as formas de intervenções mais adequadas.

No Brasil, a determinação de elementos-traço tem sido realizada por vários autores (ABREU et al., 2012; MADEIRA, 2013; PELOZATO et al., 2011; PIERANGELI et al., 2015; SOUZA et al., 2015; TAVARES et al., 2017), nos mais variados ambientes e com o emprego de diversos procedimentos técnicos, objetivando verificar a existência de contaminação por elementos-traço, bem como, avaliar a extensão dessa contaminação.

A toxicidade, a área contaminada e a escolha do método para a quantificação de determinado elemento-traço presente nos solos de uma região, são fundamentais para se estabelecerem os valores limites das concentrações permitidas pelos órgãos regulamentadores. A seleção do método deve levar em consideração a concentração da solução extratora, tempo e temperatura de extração, tempo e tipo de agitação, relação solo/solução, dentre outros, resultando na representatividade e exatidão dos resultados a serem adaptados às rotinas de laboratório, buscando praticidade e custo-benefício (ABREU et al., 2012; PIERANGELI et al., 2015).

A escolha do método a ser utilizado poderá influenciar na concentração dos elementos-traço a serem analisados, podendo os apresentar resultados discrepantes para uma mesma amostra (SOUZA et al., 2015). Outra dificuldade na comparação dos resultados experimentais consiste na adaptação de diferentes soluções extratoras aliadas às diversas condições de tempo e temperatura utilizadas nos processos.

Os principais inconvenientes que podem ocorrer são: a escolha inadequada de um reagente pode gerar extratos que danificam partes do equipamento; a extração de soluções de amostras com concentrações diferentes às dos padrões utilizados na calibração; e até mesmo, o uso excessivo de reagentes, resultando em erros na quantificação (SOUZA et al., 2015; PELOZATO et al., 2011). Portanto, ao se decompor uma amostra para análise química devemos considerar os seguintes fatores: a sua natureza, o elemento a ser determinado, a sua concentração, o método analítico e, finalmente, a precisão e exatidão desejada.

Os métodos mais comuns empregados na digestão de amostras utilizam-se de combinações de diferentes ácidos inorgânicos e elevadas temperaturas, podendo ser por meio de sistemas abertos em blocos digestores (USEPA 3050; USEPA 3050B; Nitroperclórica e Sulfúrica), ou por meio de sistemas fechados sob pressão em fornos de micro-ondas (USEPA 3051; USEPA 3051A e USEPA 3052) (USEPA, 2007). Especificamente, os métodos analíticos indicados para a preparação de amostras sólidas a serem usados na determinação de vanádio são USEPA 3050B e USEPA 3051A, estando em conformidade com a resolução CONAMA 420/2009 e com a Agência de Proteção Ambiental dos Estados Unidos (USEPA).

No método USEPA 3050B, sistema aberto, a solução extratora utilizada é a combinação de ácido nítrico mais água oxigenada $\left(\mathrm{HNO}_{3}+\mathrm{H}_{2} \mathrm{O}_{2}\right)$. Devido a praticidade e baixo custo é utilizado na maioria dos 
laboratórios, porém, é um procedimento demorado e por ser um sistema aberto pode ocasionar perdas por volatilização.

No método USEPA 3051A, sistema fechado, a solução extratora empregada é a combinação de ácido nítrico e ácido clorídrico na proporção 3:1 $\left(\mathrm{HNO}_{3}+\mathrm{HCl}\right)$ em forno de micro-ondas em condições de elevada temperatura e pressão. Por ser um sistema fechado apresenta maior dissolução da amostra, tempo reduzido de digestão e menor perda por volatilização. No entanto, possui elevado custo de aquisição e devido à complexidade sua operação exige profissionais capacitados.

Entretanto, há diferenças tanto nas terminologias quanto nos métodos de extração empregados, devido as particularidades das análises: tempo, temperatura, diferentes soluções de digestão, granulometria do solo, relação solo/solução, entre outros (PELOZATO et al., 2011; SOUZA et al., 2015). Além do mais, a variabilidade das condições de extração pode acarretar diferenças nas quantidades extraídas por um método específico (TAVARES et al., 2017). Assim sendo, é imprescindível que se compare os métodos de extração e quantificação dos elementos-traço no solo com vistas a estabelecer sua precisão e acurácia.

A comparação entre os resultados dos métodos ou modelos utilizados, por sua vez, deve ser efetuada por meio da aplicação de testes estatísticos de concordância, que verificam se esses mesmos métodos ou modelos são equivalentes a ponto de se poder substituir um pelo outro. Para que isso ocorra, os dois métodos em comparação devem apresentar um erro que não ofereça impacto significativo para que sejam permutáveis (BUSSAB et al., 2010; DEMÉTRIO et al., 2011; MILAN, 2014)

Diversos indicadores podem ser utilizados para avaliar a similaridade entre dois métodos ou modelos distintos quanto às estimativas dos valores das variáveis respostas. Dentre eles, pode-se destacar os seguintes critérios: o coeficiente de correlação linear de Pearson, $r$; o coeficiente de regressão linear, o coeficiente de determinação, $r^{2}$; o índice de concordância, $\boldsymbol{d}$, de Willmott (WILLMOTT et al., 2012), o coeficiente de confiança ou desempenho, $c$, proposto por Camargo et al. (1997) e o teste de identidade proposto por Leite et al. (2002).

Neste trabalho objetivou-se comparar os métodos de digestão de amostras USEPA 3050B e USEPA 3051A, recomendados pela legislação brasileira, para analisar o desempenho na eficiência de determinação de teores de vanádio em solos.

\section{MATERIAIS E MÉTODOS}

Os dados primários utilizados neste trabalho consistiram nos teores de vanádio quantificados por Suppi (2020), em amostras do horizonte A de solos do estado de Santa Catarina, pertencentes ao banco de solos do Departamento de Solos da Universidade do Estado de Santa Catarina. Para esta pesquisa foram selecionadas 52 amostras das classes de solos mais representativas do estado, sendo na sua maioria Argissolos, Nitossolos e Cambissolos. As amostras foram coletadas em perfis modais e em áreas sob mínima ou nenhuma atividade antrópica.

O controle de qualidade das análises foi realizado utilizando-se amostras de referência SRM 2709 San Joaquin soil (Baseline trace element concentrations) certificado pelo National Institute of Standards and 
Technology (NIST).

Inicialmente, as amostras foram postas em contato com ácido nítrico e peróxido de hidrogênio por 24 horas antes de iniciar a digestão, a fim de oxidar a matéria orgânica. A digestão das amostras foi procedida utilizando-se os métodos USEPA 3050B e USEPA 3051A.

No método USEPA 3050B, digeriu-se aproximadamente 1,0 g de amostra de solo em 10,0 $\mathrm{mL}$ de ácido nítrico em bloco digestor aberto por 10 minutos a $90 \pm 5^{\circ} \mathrm{C}$, monitorada com termômetro infravermelho digital. Após, as amostras foram resfriadas e adicionado mais 5,0 $\mathrm{mL}$ de ácido nítrico e levadas novamente ao bloco digestor para aquecer a $90 \pm 5^{\circ} \mathrm{C}$ por mais 2 horas. Em seguida, foram novamente resfriadas e adicionado 2,0 mL de água destilada e $3,0 \mathrm{~mL}$ de água oxigenada $\left(\mathrm{H}_{2} \mathrm{O}_{2}\right)$ a $30 \%$. Sendo conduzidas novamente ao bloco digestor para aquecer a $90 \pm 5^{\circ} \mathrm{C}$ por mais 2 horas. Após novo resfriamento, é adicionado $5,0 \mathrm{~mL}$ de ácido clorídrico e $10,0 \mathrm{~mL}$ de água destilada e novamente aquecida por 5 minutos a $90 \pm 5^{\circ} \mathrm{C}$. As amostras são filtradas e armazenadas em recipientes plásticos.

Para o método USEPA 3051A, digeriu-se aproximadamente $0,25 \mathrm{~g}$ de amostra de solo em $4,5 \mathrm{~mL}$ de ácido nítrico (65\% P. A.) concentrado e 1,5 mL de ácido clorídrico (37\% P. A.) em forno de micro-ondas com tubos de Teflon PFTE à pressão de $0,76 \mathrm{Mpa}$, alcançando uma temperatura aproximada de $175^{\circ} \mathrm{C}$, nas condições para abertura das amostras recomendadas pelo fabricante. Após a digestão, a amostra foi filtrada e seu volume completado até $40,0 \mathrm{~mL}$ de água destilada. O micro-ondas utilizado foi Anton PAAR Multiwave 3000 , com rotor modelo MF50.

O Limite de Detecção Qualitativo do Método Analítico (LDQM) foi calculado segundo a equação $L D Q M=F \boldsymbol{x} \times(\boldsymbol{M} \pm \boldsymbol{t} \times \boldsymbol{s}) \mathbf{2 0}$. Onde, $\boldsymbol{F d}$ é o fator de diluição, $\boldsymbol{M}$ é a média das provas em branco, $\boldsymbol{t}$ é o valor $\mathrm{t}$ Student para intervalo de confiança de 99\%, em função do número de graus de liberdade de medições repetidas e $s$ é o desvio padrão das provas em branco.

A quantificação de vanádio foi efetuada por meio de espectrometria de emissão óptica por plasma acoplado indutivamente (ICP-OES), modelo Optima 8300 da marca Perkin Elmer.

As análises estatísticas dos dados foram conduzidas utilizando-se planilha eletrônica e o software $R$ ( $R$ Core Team 2020), adotando-se nas análises confirmatórias o nível de significância de 5 \%. Inicialmente foi testada a normalidade dos dados utilizando o teste de Kolmogorov-Smirnov.

A concordância de resultados entre os métodos de digestão para abertura de amostras, USEPA 3050B e USEPA 3051A, foi avaliada com base no teste t de Student, no coeficiente de correlação de Pearson (r), o coeficiente de determinação $\left(\boldsymbol{R}^{2}\right)$, o índice de concordância (d) (WILLMOTT ET al., 2012) e o índice de confiança (c) proposto por Camargo et al. (1997). Avaliou-se a identidade analítica entre os métodos utilizouse o critério estatístico proposto por Leite et al. (2002). Para a estimativa e testes dos parâmetros do modelo de regressão linear e para a aplicação do teste $t$, adotou-se a transformação logarítmica dos dados.

O coeficiente de correlação de Pearson ( $r$ ) foi obtido por análise de regressão linear, utilizando-se o método de mínimos quadrados, extraindo-se a raiz quadrada do valor do coeficiente de determinação $\left(r^{2}\right)$. Os valores de $\boldsymbol{r}$ variam entre 0 , ausência de correlação linear, e $\mp 1$, correlação linear perfeita. 0 índice de concordância, d, proposto por Willmott et al. (2012) permite a avaliação dos desvios entre os valores obtidos 
por meio dos dois métodos. É calculado conforme a equação:

$$
\boldsymbol{d}=1-\frac{\sum_{i=1}^{n}\left(Y_{1 i}-Y_{2 i}\right)^{2}}{\sum_{i=1}^{n}\left(\left|Y_{1 i}-\overline{Y_{1 l}}\right|+\left|Y_{2 i}-\overline{Y_{2 l}}\right|\right)^{2}}
$$

Onde, $\boldsymbol{d}$ é o índice de concordância, $\mathrm{n}$ é o número de pares de dados, $\boldsymbol{Y}_{1 i}$ é o i-ésimo valor da leitura com o método USEPA 3050B, $\boldsymbol{Y}_{2 i}$ é o i-ésimo valor da leitura com o método USEPA 3051A, $\overline{\boldsymbol{Y}_{1 \imath}}$ é a média dos valores de $\boldsymbol{Y}_{1 i} \mathrm{e} \overline{\boldsymbol{Y}_{2 l}}$ é a média dos valores de $\boldsymbol{Y}_{2 i}$. Os valores de $\boldsymbol{d}$ variam de 0 , nenhuma concordância, a 1, concordância perfeita.

O índice de confiança, $\boldsymbol{c}$, adimensional, proposto por Camargo et al. (1997) expresso pela equação:

$$
\boldsymbol{c}=\boldsymbol{r} * \boldsymbol{d}
$$

Conforme os autores $c$ é interpretado como: Ótimo ( $c>0,85)$; muito bom $(0,76 \leq c \leq 0,85)$; bom $(0,66$ $\leq c \leq 0,75)$; mediano $(0,61 \leq c \leq 0,65)$; sofrível $(0,51 \leq c \leq 0,60)$; mau $(0,41 \leq c \leq 0,50)$; péssimo $(c<0,40)$.

O teste de identidade conforme Leite et al. (2002), trata-se de uma regra de decisão referente à hipótese de identidade entre dois vetores quantitativos. Segundo os autores, o uso do coeficiente de correlação linear, isoladamente, não é suficiente para decidir sobre a identidade entre dois métodos analíticos, devido à possibilidade do intercepto e do coeficiente de regressão serem diferentes de 0 e 1 , respectivamente. Dois métodos que se correlacionem de maneira perfeita produzirão um modelo no qual os coeficientes assumirão os seguintes valores: $\beta_{0}=0$ e $\beta_{1}=1$. Portanto, é possível realizar um teste de hipótese baseado nessas premissas de maneira a avaliar a significância dos coeficientes do modelo. 0 procedimento proposto pelos autores, resultante da combinação entre a estatística F modificada, o teste do erro médio e o coeficiente de correlação linear, é eficiente e não subjetivo, e é recomendado para se testar a identidade entre quaisquer dois métodos analíticos ou quaisquer dois vetores de dados quantitativos.

\section{RESULTADOS E DISCUSSÃO}

Os teores médios de vanádio recuperados nas amostras de referência SRM 2709 San Joaquin soil pelos métodos USEPA 3051A e USEPA 3050B foram de 93,50 \% e 69,10 \%, respectivamente. Este comportamento pode ser atribuído à composição da solução extratora e ao uso de ambiente fechado no método USEPA3051A que propiciou uma digestão mais completa.

Os teores de vanádio obtidos nas amostras de solos (Tabela 1) pelo método USEPA 3051A foram superiores $(P<0,05)$ àqueles obtidos com o método USEPA 3050B. corroborando o comportamento observado nas amostras de referência SRM 2709 San Joaquin soil. Ambos os métodos possuem um grau de dispersão similar, indicando o mesmo grau de confiabilidade analítica na determinação dos teores de vanádio nos solos. A maior capacidade de determinação de vanádio pelo método USEPA 3051A, observada principalmente em amostras de solos com elevada concentração do metal, pode ser atribuída ao efeito combinado da pressão e da alta temperatura de ebulição atingida, que aumentam a cinética de reação e, consequentemente, diminuem o tempo de digestão. Considerando que a temperatura é uma das variáveis que mais influenciam na eficiência da digestão da amostra, o seu controle permite maior reprodutibilidade dos resultados. Deve-se ainda, tomar em conta o fato do método USEPA 3050B ser realizado em sistema aberto e sob pressão atmosférica quando comparado ao sistema fechado em micro-ondas utilizado no método USEPA 3051A, bem como a possibilidade de perdas do analito por volatilização no primeiro método 
(CAMPOS et al., 2005; SILVA, 2012; SOUZA et al., 2015).

As estimativas dos parâmetros de regressão linear obtidos foram, $\boldsymbol{B}_{\boldsymbol{0}}=-\mathbf{0 , 5 1 5 9}$ e $\boldsymbol{B}_{\boldsymbol{1}}=0,5885$ (Figura 1). O valor do intercepto $\left(\boldsymbol{B}_{0}\right)$ não diferiu de zero $(P>0,05)$ indicando que os dois métodos não diferem na origem, no entanto, a inclinação $\left(\boldsymbol{B}_{1}\right)$ diferiu de um $(P<0,05)$ indicando um comportamento diferente dos dois métodos nos maiores teores de vanádio no solo. No caso do teste de identidade, de acordo com os critérios propostos por Leite et al. (2002), o valor $\mathrm{F}\left(\mathrm{H}_{0}\right)$ foi significativo $(580,1 ; \mathrm{P}<0,05)$, assim como o erro médio calculado $\left(t_{\bar{e}}\right)$ diferiu de zero $(\mathrm{P}<0,05)$. Isto ocorre, principalmente, quando o valor do quadrado médio do erro é pequeno, comparativamente ao quadrado médio da regressão, resultando num valor de $\mathrm{F}\left(\mathrm{H}_{0}\right)$ significativo, o que indica a diferença na capacidade extrativa entre os dois métodos.

Tabela 1: Estatísticas descritivas dos teores de vanádio nos solos.

\begin{tabular}{|c|c|c|}
\hline Estatísticas & USEPA3051A & USEPA3050B \\
\hline Média $\left(\mathrm{mg} \mathrm{kg}^{-1}\right)$ & 190.91 & 111.84 \\
\hline Mediana $\left(\mathrm{mg} \mathrm{kg}^{-1}\right)$ & 82.81 & 46.24 \\
\hline Desvio padrão (mg kg-1) & 200.98 & 123.27 \\
\hline Coeficiente de Variação (\%) & 105,27 & 110,22 \\
\hline Curtose & -0.44 & -0.50 \\
\hline Assimetria & 0.97 & 0.94 \\
\hline Mínimo $\left(\mathrm{mg} \mathrm{kg}^{-1}\right)$ & 3.97 & 0.86 \\
\hline Máximo $\left(\mathrm{mg} \mathrm{kg}^{-1}\right)$ & 652.23 & 392.23 \\
\hline Amostra (n) & 52 & 52 \\
\hline
\end{tabular}

Portanto, com base na regra de decisão adotada, $Y_{j} \neq Y_{1},\left(Y_{j}=\right.$ USEPA 3050B e $Y_{1}=$ USEPA 3051A), a hipótese $\mathrm{H}_{0}: \beta=[0,1]$ deve ser rejeitada, assumindo-se que há diferença entre os métodos na capacidade de extração de vanádio das amostras de solos.

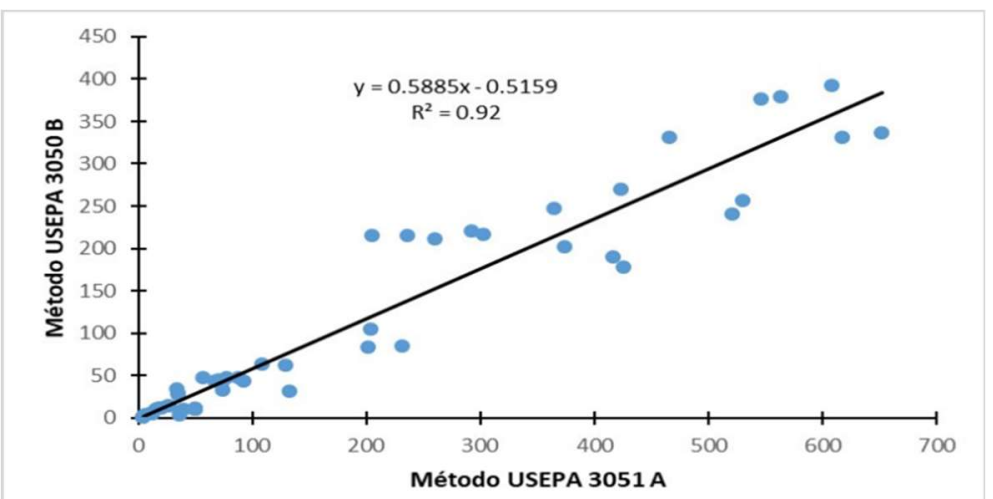

Figura 1: Relação entre os teores de vanádio recuperados pelos métodos USEPA 3050B e USEPA 3051A das amostras nos solos $\left(\mathrm{mg} \cdot \mathrm{Kg}^{-1}\right)$.

Silva (2012), comparando a eficiência dos dois métodos na extração de teores considerados ambientalmente disponíveis de $\mathrm{Zn}, \mathrm{Cu}, \mathrm{Cd}, \mathrm{Pb}$ e $\mathrm{Ni}$, obteve altas correlações positivas entre os métodos USEPA 3051A e USEPA 3050B. A autora considerou o primeiro método mais eficiente que o segundo, pois além de proporcionar maior recuperação, exige menor tempo de digestão, menor consumo de solução extratora e reduz o risco de contaminação. Madeira (2013), comparando os métodos USEPA 3051A e USEPA 3050B na determinação de teores naturais de $\mathrm{Ni}$ em solos catarinenses, verificou que o método USEPA 3051A apresentou taxas de recuperação dos teores de Ni nas amostras de referência superiores ao método USEPA 3050B, devido ao procedimento ser conduzido em sistema fechado, em micro-ondas, apresentando 
vantagens como o tempo de digestão, altas temperaturas mantidas homogêneas em todas as amostras e a elevada pressão que minimiza as perdas dos analitos. Entretanto, Campos et al. (2005) observaram que não houve diferença entre os métodos USEPA 3051A e USEPA 3050B na extração de $\mathrm{Cr}$, Cu, Ni e Zn em fosfatos de rocha. Para Pelozato et al. (2011) o método USEPA 3050B apresentou potencial para ser utilizado como método padrão na extração de cádmio, cobre e zinco de solos catarinenses derivados de basalto e granitomigmatito. Souza et al. (2012) verificaram que os métodos USEPA 3051A e USEPA 3050B são idênticos na extração de teores de Zn e Cu em fertilizantes minerais e materiais secundários.

Conforme Zonta et al. (2019, In: ZUFFO 2019), ambos os métodos, USEPA 3051A e USEPA 3050B, são comumente empregados na digestão para abertura de amostras de solos, devendo a escolha do método levar em consideração as condições específicas de cada material, como seu tipo e origem.

De modo geral, a escolha deve ser analisada com cautela, tanto em relação ao uso de soluções extratoras e ao tipo de abertura mais adequado para determinação de elementos-traço em solos e plantas, quanto em relação a maior reprodutividade analítica no controle de qualidade, considerando-se, além da segurança operacional, os custos de implantação e manutenção.

\section{CONCLUSÔES}

O método USEPA 3051A foi mais eficiente que o método USEPA 3050B na recuperação de vanádio nas amostras de referência de referência SRM 2709 San Joaquin soil. Os teores de vanádio obtidos nos solos com o método USEPA 3051A foram maiores que aqueles determinados com o método USEPA 3050B, principalmente nas amostras com maiores concentrações do elemento. Embora não tenha sido observada identidade entre os métodos conforme o critério de Leite et al. (2002), os valores dos coeficientes, de correlação linear e de determinação, e os índices, de concordância e de confiança, obtidos possibilitam a utilização de ambos os métodos.

\section{REFERÊNCIAS}

ABREU, L. B.; ARAUJO, E. F.; CURI, N.; MARQUES, J. J. Determinação do teor total de elementos-traço em solos sob eucalipto na depressão central, Rio Grande do Sul. Revista brasileira de Agrociência, v.18, n.1, p.33-43, 2012.

BUSSAB, W. D. O.; MORETTIN, P. A.. Estatística Básica. São Paulo: Saraiva, 2010.

CAMARGO, A. P.; SENTELHAS, P. C.. Avaliação do desempenho de diferentes métodos de estimativa da evapotranspiração potencial no Estado de São Paulo. Revista Brasileira de Agrometeorologia, Santa Maria, v.5, n.1, p.8997, 1997.

CAMPOS, M. L.; SILVA, F. N.; FURTINI NETO, A. E.; GUILHERME, L. R. G.; MARQUES, J. J.; ANTUNES, A. S.. Determinação de cádmio, cobre, cromo, níquel, chumbo e zinco em fosfatos de rocha. Pesquisa Agropecuária Brasileira, v.40, n.4, p.361-367, 2005. valores orientadores de qualidade do solo quanto à presença de substâncias químicas e estabelece diretrizes para o gerenciamento ambiental de áreas contaminadas por essas substâncias em decorrência de atividades antrópicas. Brasília: CONAMA, 2009.

DEMÉTRIO, C. G. B.; ZOCCHI, S. S.. Modelos de Regressão. Piracicaba, 2011.

LEITE, H. G.; OLIVEIRA, F. H. T.. Statistical procedure to test the identity of analytical methods. Communications in Soil Science and Plant Analysis, v.33, p.1105-1118, 2002.

MADEIRA, M. M.. Determinação dos teores naturais de níquel em solos do estado de Santa Catarina. (Dissertação de Mestrado) - Universidade do Estado de Santa Catarina, Lages, 2013.

MILAN, L. A.. Estatística Aplicada. São Carlos: UFSCAR, 2014. PELOZATO, M.; HUGEN, C.; CAMPOS, M. L.; ALMEIDA, J. A.; SILVEIRA, C. B.; MIQUELLUTI, D. J.; SOUZA, M. C.. 
Comparação entre métodos de extração de cádmio, cobre e zinco de solos catarinenses derivados de basalto e granitomigmatito. Revista de Ciências Agroveterinárias, Lages, v.10, n.1, p.54-61, 2011.

PIERANGELI, M. A. P.; GUILHEME, L. R. G.; CARVALHO, G. S.; CARVALHO, C. A.; SILVA, C. A.; PIERANGELI, L. M. P..

Elementos-traço em áreas de vegetação nativa e agricultura intensiva do estado de Mato Grosso determinados por Fluorescência de Raios-X por Reflexão Total. Revista Brasileira de Ciência do Solo, v.39, n.4, p.1048-1057, 2015.

SILVA, Y. J. A. B.. Métodos de digestão de solos e fertilizantes para análises de metais pesados. (Dissertação de Mestrado) - Universidade Federal Rural de Pernambuco, Recife, 2012.

SOUZA, V. L. B.; LIMA, V.; HAZIM, C. A.; FONSECA, C. K. L.; SANTOS, S. O.. Biodisponibilidade de metais traço em sedimentos: uma revisão. In: BRAZILIAM JOURNAL OF RADIATION SCIENCES. INTERNATIONAL JOINT CONFERENCE RADIO, BRAZIL. SOCIEDADE BRASILEIRA DE PROTEÇÃO RADIOLÓGICA - SBPR. Anais. Gramado, 2015. p.26-29.

SUPPI, I. M.. Teores de Vanádio em solos. Destinatário:
Marcos Roberto Schein. Lages, 2020.

TAVARES, S. R. L.; OLIVEIRA, S. A.. Avaliação de diferentes métodos de extração de metais pesados em solos contaminados provenientes de atividades de galvanoplastia. Rio de Janeiro: Embrapa Solos, 2017.

USEPA. U. S. Environmental Protection Agency. Method 3050B: Acid digestion of sediments, sludges, and soils. Washington: USEPA, 1996.

USEPA. U. S. Environmental Protection Agency. Method 3051A: Microwave assisted acid digestion of sediments, sludges, soils, and oils. Washington: USEPA, 2007.

WILLMOTT, C. J.; ROBESONB, S. M.; MATSUURA, K.. Short Communication: A Refined Index of Model Performance. Intl. Journal of Climate, Maden, v.32, p.2088-2094, 2012.

ZONTA, E.; SANTOS, F. S.; RIBEIRO, J. C.; SOBRINHO, N. M. B A.. Comparação entre os principais métodos de digestão para a determinação de metais pesados em solos e plantas. In: ZUFFO, A. M.. A produção do conhecimento nas ciências agrarias e ambientais 2. Ponta Grossa: Antena, 2019.

A CBPC - Companhia Brasileira de Produção Científica (CNPJ: 11.221.422/0001-03) detém os direitos materiais desta publicação. Os direitos referem-se à publicação do trabalho em qualquer parte do mundo, incluindo os direitos às renovaç̃oes, expansões e disseminações da contribuiç̃o, bem como outros direitos subsidiários. Todos os trabalhos publicados eletronicamente poderão posteriormente ser publicados em coletâneas impressas sob coordenação da Sustenere Publishing, da Companhia Brasileira de Produção Científica e seus parceiros autorizados. Os (as) autores (as) preservam os direitos autorais, mas não têm permissão para a publicação da contribuição em outro meio, impresso ou digital, em português ou em tradução. 